\title{
Laboreal
}

Volume $7 \mathrm{~N}^{\circ} 2$ | 2011

Varia

\section{Contribución a una introducción al estudio de los sistemas humanos-maquina}

Contributo para uma introdução ao estudo dos sistemas homens-máquinas Contribution à une introduction aux études des systèmes hommes-machine Contribution to an introduction to the study of man-machine systems

\section{Michel Olivier}

Traductor. Martín Walter

\section{OpenEdition}

\section{Journals}

Edición electrónica

URL: http://journals.openedition.org/laboreal/7866

DOI: $10.4000 /$ laboreal.7866

ISSN: 1646-5237

Editor

Universidade do Porto

Referencia electrónica

Michel Olivier, "Contribución a una introducción al estudio de los sistemas humanos-maquina »,

Laboreal [En línea], Volume 7 №2 | 2011, Publicado el 01 noviembre 2011, consultado el 24 septiembre 2020. URL : http://journals.openedition.org/laboreal/7866 ; DOI : https://doi.org/10.4000/laboreal. 7866

Este documento fue generado automáticamente el 24 septiembre 2020

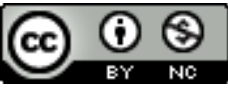

Laboreal está licenciado com uma Licença Creative Commons - Atribuição-NãoComercial 4.0 Internacional. 


\title{
Contribución a una introducción al estudio de los sistemas humanos- maquina
}

\author{
Contributo para uma introdução ao estudo dos sistemas homens-máquinas \\ Contribution à une introduction aux études des systèmes hommes-machine \\ Contribution to an introduction to the study of man-machine systems
}

\section{Michel Olivier}

Tradución : Martín Walter

\section{REFERENCIA}

Artigo original : Olivier, M. (1967). Contribution à une introduction aux études des systèmes hommes-machine. Bulletin du C.E.R.P., 1967-XVI-nº1, p. 61-72.

1 Es de uso que una introducción sea el resultado de una reflexión retrospectiva; que esta preceda el cuerpo principal del tema se debe a un simple artificio de presentación. Proceder de forma inversa refiere darle al término "introducción" en un sentido prospectivo. Es marcar los rasgos generales del edificio sin la presunción de quién pronuncia un discurso tras la instalación del primer ladrillo, sino con la sana desconfianza del constructor que revisa los materiales disponibles. En el caso de los estudios llamados "de sistemas" la necesidad de tal aproximación es aún más importante, dado que los materiales y herramientas que deberemos utilizar son demasiado seductores al punto de ser sospechosos y que el campo en el que deberemos construir tiene límites fijados arbitrariamente, ocupados por disciplinas tan variadas (biología, economía, psicología, sociología) que llevan a pensar en una Babel. Sería oportuno en este contexto recurrir al psicoanálisis del conocimiento objetivo preconizado por $\mathrm{G}$. Bachelard y estudiar desde esta perspectiva la noción de sistema y de conceptos relacionados. Esto se sale del dominio típico de una introducción. 
2 Para estudiar un sistema, es decir un objeto demasiado grande o complejo para su observación completa o experimentación, se utilizan conceptos generales sobre este tipo de entidad, como primer bosquejo de un modelo práctico. No carece de interés recordar cómo, hace alrededor de diez años, el sociólogo americano P. Meadows (1957) realizó una exposición sobre este tema: " Tenemos la impresión aún si no somos más que un lector ocasional de los trabajos de investigación actuales de que la palabra "modelo" es el fin del fin del lenguaje científico (...). En relación con el concepto de "sistema," también de moda, un "modelo" ha devenido en un instrumento poderoso y prestigioso". De hecho, una revisión de la literatura demuestra que distintos modelos de sistemas mantienen semblanza con una vaga analogía, usualmente mal definida aunque a veces poética, o de empréstitos de la física de leyes cuya aplicación en el ámbito de las ciencias humanas demandaría al menos una cierta prudencia, o de una matematización refinada aparentemente prematura a juzgar por sus dificultades en poner en relieve lo interesante. Si bien es indiscutible que una formalización es indispensable para la constitución de bases teóricas suficientes a partir de las cuales derivar aplicaciones, no es por ello menos indiscutible de que son aún insatisfactorias, sobre todo en el ámbito de las descripciones morfológicas o históricas de sistemas, ya que carecen de suficiente coherencia.

3 Podríamos preguntarnos entonces por la utilidad de introducir la noción de sistema en el análisis de conjuntos hombre-máquina o más generalmente en aquella de los conjuntos tecnológicos complejos, productos de la transformación del hombre del mundo natural y social. La idea es que la ergonomía, la adaptación del trabajo al hombre, ha llegado a un punto de saturación especulativa. Más precisamente, parece que las soluciones técnicas aportadas a los problemas del hombre en el trabajo por los estudios de las actividades motrices o de la toma y tratamiento de la información, o mismo de algunos estudios pico-sociológicos, son esencialmente limitados en el tiempo porque la evolución industrial los vuelve rápidamente efímeros y en el espacio porque es reconocible que aún si se ha reforzado un eslabón de la cadena, la perjuiciosa tensión ejercida sobre su totalidad persiste todavía. Esto no significa que la extensión de estos estudios haya llegado a su saturación -al menos a mediano plazo puesto que su interés no ha sido desafiado- pero indica la necesidad de superación y de un esfuerzo de adaptación. Notamos que es posible extraer valor de la proliferación de trabajos sobre este tema, y que la combinación de la idea de sistema junto con los problemas que plantea la integración de hombres en estos conjuntos tecnológicos complejos a dado nacimiento a un fructuoso campo de investigaciones que permiten esta superación.

Que lo que prosigue sea entonces considerado como el resultado provisorio y parcial de un trabajo más colectivo que individual, como el emplazamiento de elementos de un problema y de una tentativa de aunar distintos conceptos en favor de la elaboración de un sistema.

\section{Revisión esquemática de diversas concepciones del estudio de sistemas.}

Ante todo, se trata de esbozar un rápido cuadro histórico de los distintos "movimientos" dedicados al estudio de sistemas pertinentes a nuestro tema, para ubicarlos en nuestro cuadro analítico general de los sistemas hombre-máquina. 


\section{La teoría general de sistemas}

6 El movimiento de la "general systems theory" nace -en la medida en que es posible hablar de nacimiento ex nihilo- al fin de la entre-guerra en Viena, a partir de trabajos del biólogo y filósofo L. von Bertalanffy sin publicar o poco publicitados hasta el fin de la segunda guerra mundial (von Bertalanffy, 1945, 1949).

7 Teórico de concepciones organicistas en biología, L. von Bertalanffy (1950a) extendió sus ideas respecto de una gestalt orgánica a una teoría general de sistemas debiendo realizar la unidad de la ciencia, basada en la estructura isomorfa de las leyes en sus distintas ramas. No se puede negar que este movimiento tenga origen en las mismas tendencias que aquellas que precedieron la apertura de la teoría de la Gestalt, y el mismo anti-mecanismo, el mismo anti-atomismo, el mismo esquema hilemórfico del naturalismo alemán, los mismos principios de totalidad, de organización, de interacción dinámica. Es difícil no conectar las concepciones de L. von Bertalenffy con las de K. Goldstein (1934). Su consideración de las analogías entre las distintas ramas de la ciencia tiene sus orígenes en la fenomenología física de E. Mach (1910).

8 La reunión en torno de estas ideas por parte de autores de horizontes diversos como A. Rapoport, D. Kreck, R. W. Gérard, por ejemplo, tras su instalación al otro lado del Atlántico (Canada, 1949 ; USA, 1954) y el apogeo de sus concepciones en la fundación de la Society for the Advancement of General Systems Theory en 1954, seguida de la publicación anual a partir de 1956 de "General Systems," de la cual es coeditor con A. Rapoport, no debe disimular el hecho de que nos encontramos aún lejos de una teoría general de sistemas y nos vemos en el compromiso de aceptar la existencia de dos perspectivas para el estudio de sistemas. Para dar cuenta de la teoría de L. von Bertalanffy, es inútil agregar las reformulaciones que se han propuesto, así como las críticas que se le han realizado, y podemos contentarnos con la breve mención de algunos de sus principales aspectos. Un sistema, conjunto de elementos en interacción, puede ser representado en forma abstracta mediante un sistema de ecuaciones diferenciales que utilizan el tiempo como variable independiente; la introducción de condiciones específicas concernientes a los parámetros y las variables permite mostrar el isomorfismo estructural entre diversos fenómenos biológicos, físicos, económicos y otros. Por otra parte permite ilustrar más adecuadamente ciertas características de sistemas orgánicos. En primer lugar, la consideración de dos casos extremos, uno en el que el cambio de cada variable depende solamente de sí misma y de otro en el que el cambio de cada variable determina un cambio en todas las otras y afecta, por lo tanto el sistema en su conjunto, permite contraponer una hipótesis mecanicista de independencia entre los elementos cuya variación agregada corresponde a la del conjunto global, lo que justifica el estudio de partes aisladas, y de la hipótesis gestaltista según la cual el sistema se considera como un todo al interior del cual cada elemento no tiene sentido y existencia sino a través de su interacción con otros elementos. En segundo lugar, podemos caracterizar de este modo organismos por la segregación o mecanización progresiva, es decir por la reducción de la interacción entre los elementos en el tiempo y su ordenamiento en subsistemas subordinados, que implican el crecimiento de la complejidad del sistema y una regulación más y más difícil. Esta segregación progresiva se ve compensada por una centralización progresiva, cuya evolución depende principalmente del tiempo. Un estudio de las partes puede 
realizarse solamente desde el nivel de sistemas altamente mecanizados, subdivididos en subconjuntos altamente especializados.

9 L. von Bertalanffy (1959b) introdujo entonces las nociones de sistema abierto y de equilibrio dinámico, nociones recuperadas de la termodinámica de fenómenos irreversibles, que le permitieron interpretar otras características de sistemas orgánicos. Un sistema es abierto cuando existe un intercambio material con el ambiente, un equilibrio dinámico es el estado de tal sistema mediante el cual todas las variables macroscópicas se mantienen constantes aunque exista un cambio en los procesos de flujo de substancias. En el caso de los fenómenos vitales, este estado de equilibrio final es equifinal, es independiente de las condiciones iniciales, es decir que puede ser el resultado de distintos estados y de distintas vías dado que está determinado únicamente por los parámetros del sistema y del proceso de intercambio de substancias con el medio ambiente. La equifinalidad es responsable de las regulaciones preliminares de sistemas orgánicos y se ve restringida progresivamente por la subdivisión del sistema en partes separadas. En otros términos, puede decirse que se mantiene el equilibrio dinámico de la estructura diferenciada del organismo en el seno de un medio exterior menos organizado y que este puede alcanzarse por distintos modos de equifinalidad

\section{La teoría de los sistemas auto-organizados}

Otra forma de examinar la teoría de los sistemas es la cibernética, más precisamente mediante el enfoque de W. R. Ashby (1956). Este segundo movimiento, del que pueden citarse otros representantes como S. Beer y G. Pask, se ha establecido con muchas reticencias y contactos con el primer grupo, sobre la base de las primeras contribuciones de W. R. Ashby (1947) que reivindica una parte importante de la teoría de los sistemas mediante la introducción del concepto de auto-organización ( Yovits \& S. Cameron, 1960 ; von Foerster \& Zopf, 1962). Se sabe que la cibernética estudia la dirección de sistemas dinámicos complejos compuestos de subsistemas más simples interactuantes cuyo estado se caracteriza mediante los valores de parámetros y de derivados según el tiempo de elementos constitutivos. Se ocupa entonces, en particular, de la acción ejercida sobre estos parámetros, acción que modifica el proceso o pasaje de un estado a otro. Debemos considerar que los problemas de control y de comunicación sobre los cuales hace hincapié constituyen solo un aspecto parcial del estudio de sistemas, y es solamente cuando esta introduce la noción de organización, de estructura, que esta se hace lugar en el cuadro analítico más general.

11 En este contexto, el sistema puede estudiarse como un todo inseparable, una caja negra, mediante el análisis de sus estados de entrada y salida, o en tanto un conjunto de componentes funcionales que definen su estado. Si especificamos un conjunto $\mathrm{S}$ de estados del sistema, el conjunto I de estados del medio ambiente o de entrada, y si S es el producto cartesiano \{i\} Ti en el que cada parte i se caracteriza por el conjunto de estados $\mathrm{T}_{\mathrm{i}}$, su organización se define por la aplicación $\mathrm{f}$ del producto I x S en $\mathrm{S}$ (Ashby, 1956). Tenemos entonces una relación, un ordenamiento de estructuras que controlan o dirigen el proceso para que este alcance algún objetivo.

El concepto de auto-organización se relaciona con la capacidad de cambio cualitativo de un sistema cuando este no logra hacer frente a una perturbación, lo que W. R. Ashby denomina ultraestabilidad. Desde una mirada más global, existe multiestabilidad, 
adaptación al medio ambiente por sistemas ultraestables. La auto-organización pueden comprenderse según dos acepciones bien distintas, como paso de la no-organización a la organización o como de lo menos organizado a lo más organizado, el refuerzo de la estructura o la creación de nuevas relaciones ya sea como el paso de una mala organización a una mejor organización por la conexión de perturbaciones externas y el objetivo del sistema. En el primer caso, considerado por G. Pask (1959), se plantea el caso contrario a la segregación progresiva, lo que podríamos llamar la sistematización o concretización, el aumento de la interacción de los elementos en el tiempo; en el segundo caso, tratado inicialmente por W. R: Ashby, existe adaptación por ensayo y error, es decir que a partir de un nivel crítico, se efectúa un salto en las características del sistema que modifica el proceso pretérito, de forma recurrente a fin de restablecer el comportamiento adecuado. Bajo esta última acepción, ninguna máquina en el sentido sugerido por W. R. Ashby puede auto-organizarse, el paso de una mala organización a una buena organización depende de un agente exterior asociado al sistema y que no puede surgir de una causa interna. De hecho, este modelo es el de un "cerebro," aunque sin mención al aprendizaje, sin modificación de las estructuras por la experiencia. Todo ocurre como si el sistema reaccionara a la perturbación actual y se transformase en otro sistema. Es en este sentido que otros autores han buscado desarrollar el concepto de auto-organización, atacando desde distintos ángulos el problema de un sistema que constituye un reflejo interno del medio ambiente, al que utiliza y mejora. De este modo, por ejemplo S. Beer (1961) propone una teoría del funcionamiento de un "cerebro" capaz de organizar, de controlar y de hacer evolucionar un sistema industrial. Es del mismo modo la razón por la cual vemos que los simposios sobre sistemas autoorganizados reúnen a especialistas de mecanismos de aprendizaje de autómatas, de la biología del cerebro, de la lógica de las neuronas, de máquinas heurísticas...

\section{La tecnología de los sistemas}

13 La ingeniería de Sistemas ("system engineering") nace de las demandas crecientes de la tecnología moderna y de la experiencia resultante de los problemas de los sistemas militares complejos adquirida durante la segunda guerra mundial. Armados con la ausencia de prejuicio de los tecnócratas, los practicantes del system engineering reciben con el mismo interés las dos tendencias mencionadas previamente, esperando que de las concepciones "filosóficas" de estos teóricos pueda emerger alguna aplicación práctica (Eckman, 1961 ; Mesarovic, 1964).

De hecho, es relativamente erróneo circunscribir el campo de actividad del ingeniero en sistemas que en su afán de análisis o de síntesis utiliza a su vez los métodos de la investigación operacional y los procedimientos de control automatizado. Si se define el proceso de un sistema como una seguidilla de operaciones o de acciones que transforman ciertas cantidades (entradas) en otras (salidas), parecería que el objetivo de la ingeniería de sistemas es la maximización o la minimización de algún valor de las salidas, como por ejemplo la eficacia o el costo. El objetivo del ingeniero de sistemas es entonces la optimización de las funciones del conjunto de acuerdo al énfasis acordado a los objetivos (Morton, 1959).

Estas dos perspectivas de la tecnología de sistemas que intitularemos respectivamente operacional y funcional se encuentran siempre íntimamente relacionadas y de forma más global puede decirse que el problema fundamental es el control y la optimización 
del desempeño del sistema. Un primer aspecto importante de la ingeniería de sistemas es la amplificación de técnicas tradicionales de investigación operacional, la definición de dos características de conjuntos técnicos complejos relacionados con su disponibilidad ("availability") ; la fiabilidad ("reliability") indicada por el índice de frecuencia de la averías, de los incidentes y de las disfunciones -las funciones principales de un sistema requieren el mantenimiento preventivo (averías por uso) y el control previo a la puesta en funcionamiento (averías infantiles)- lo que presenta, a nivel de los procesos, el problema de la estructuración de las relaciones cualitativas entre las fiabilidades de los componentes para asegurar la del sistema en su conjunto ; la mantenibilidad ("maintenability") cuyo índice es el tiempo de reparación, de puesta en funcionamiento normal, que mejora en tanto las disposiciones que lo permiten son más eficaces, es decir que las disfunciones son detectadas, localizadas y corregidas rápidamente (control, mantenimiento correctivo, aprovisionamiento de repuestos, check-lists). La distinción entre confiabilidad y mantenibilidad no es siempre tan clara en la literatura, el vínculo estrecho que existe entre estos dos parámetros caracterizan la supervivencia de un sistema (Barlow \& Proschan, 1965 ; Goldman \& Slattery, 1964).

En particular, la noción de sistema auto-recuperable o auto-reparable pone en manifiesto las dificultades propias a la distinción entre un sistema cuya fiabilidad aumenta por la introducción de redundancias, por la duplicación de componentes (o mediante su plurifuncionalidad), y un sistema cuya capacidad de recuperación aumenta gracias a elementos de socorro que necesitan dispositivos de detección de averías y de commutación entre el órgano principal y el órgano auxiliar.

17 Un segundo aspecto importante de la ingeniería de sistemas se debe al desarrollo de teorías sobre el control, que ponen en relieve los problemas de los sistemas no-lineales, a los sistemas complejos múlti-variados y al control adaptativo. En particular, este último aspecto parecería tener la mayor chance de influenciar el futuro de la ergonomía de procesos industriales. De la misma forma en que se ha propuesto el análisis del trabajo del hombre en términos de modelos de regulación simple (Faverge et al., 1966), todo parece indicar que aumentará el estudio del trabajo del operador humano desde la perspectiva de la teoría de los sistemas de control adaptativo u óptimo, que ajusta del resultado expresado en términos de un índice de performance a optimizar sus parámetros, o su estructura en respuesta a un cambio de la señal de entrada, o de un cambio espontáneo del sistema (Timpe, 1966). Es interesante hacer hincapié en la diferencia que existe entre un sistema adaptativo y un sistema de autoorganización, en un primer caso la adaptación es transitoria lo que implica en particular que toda información necesaria para el reconocimiento de perturbaciones y del programa de respuestas debe incluirse en el sistema, en el segundo caso, es permanente, existe un dispositivo de aprendizaje adaptativo, es la experiencia propia al sistema.

\section{Características generales de un sistema y particularización de los sistemas hombre-máquina.}

La noción de sistema, tal y como se desprende de este breve e incompleto repaso, de los trabajos de L. von Bertalanffy y de W. R. Ashby y de otros teóricos de sistemas, es un esquema que busca la descripción universal y homogénea. En el caso que nos ocupa, el de los conjuntos tecnológicos complejos, el objetivo debe ser un poco más modesto y se 
pretende un esquema más específico y relativamente autónomo como es de la ingeniería de sistemas. No obstante, se impone cierta distancia si se pretende aplicar este modelo a un contexto más amplio que incluye grandes conjuntos compuestos de hombres, grupos sociales, calculadoras, máquinas y equipos automatizados. Por otra parte, si se admite que cada sistema es único y particular por razones de origen, esto implica que los términos y los conceptos utilizados deben aplicarse a cada caso. Las condiciones de una síntesis se enuncian entonces de la siguiente manera : en presencia de modelos más o menos específicos e inconsistentes entre sí,

- puede considerárselos como representativos de una parte del modelo general a elaborar y ubicarse a nivel de reformulación que tenga en cuenta un mayor número de aspectos coherentes con ellos, lo que permite la extensión de la teoría, pero no su modificación (Miller, 1965) ;

- pueden situarse en un plano suficientemente abstracto para tener en cuenta los aspectos más centrales de estos modelos pero se pierde en información lo que se gana en la posibilidades de reestructuración (Mesarovic, 1963).

19 Nos ubicaremos más cercanos al primer caso, lo que nos permitirá algunas interpretaciones verbales, aunque utilizaremos el nivel de generalidad propuesto por el segundo caso, basados en los desarrollos de M. D. Mesarovic.

\section{Definición de un sistema}

20 El principio de base de la noción de sistema es el de relaciones entre objetos componentes de un conjunto que hace que este sea considerado como una entidad y no como la "suma" de elementos heterogéneos. Sea una familia de conjuntos $\mathrm{X}_{1}, \mathrm{X}_{2}, \ldots, \mathrm{X}_{\mathrm{j}}$, .... $X_{n}$, cada conjunto caracteriza un objeto, un sistema es una relación $R=\Pi_{j} X_{j}$ de esta familia de conjuntos. La introducción de un proceso que permite definir inductivamente los términos $\mathrm{Xj}$ y $\mathrm{Rj}$ especifica completamente un sistema. Para un conjunto de objetos dado, las relaciones consideradas dependen del punto de vista. Esta definición general puede parecer general y abstracta, veremos a continuación que otorga los medios necesarios para la enunciación más estricta de distintas modalidades de estudio de conjuntos tecnológicos complejos porque permite hacer referencia a una clase de modelo en los que la interacción está formalmente determinada.

\section{Limites de un sistema}

21 Un sistema tiene límites arbitrarios, la división del universo de un sistema S y de un ambiente $\mathrm{E}$ es parte de una convención, siempre es posible extender el campo de estudio estimando la existencia de un sistema S' que incluye S. En ese caso, $S$ es un subsistema de S'. El sistema S' es la unión de S y de una parte de E. En este sentido, es importante notar que la distinción entre un sistema abierto y un sistema cerrado es puramente teórica, al menos en lo que nos concierne. Un conjunto tecnológico complejo es abierto porque en su análisis se ignora cierto número de sus componentes, generando por ejemplo la hipótesis que cierta amortización se debe a influencias externas ejercidas sobre el sistema. No obstante, el hecho de que existan incertitudes en este sistema incompleto presenta recurrentemente el problema de la decisión y del control. 


\section{Reticulación del sistema en componentes} una relación más abstracta que es la estructura del sistema. Más precisamente, distinguimos dos vías para el estudio de estructuras de un conjunto tecnológico complejo :

a. El análisis de la estructura operacional de un sistema en el que se describe un conjunto de causas y efectos en términos de relaciones entradas-salidas entre componentes, se sitúa a nivel del proceso de un sistema.

b. El análisis de la estructura funcional en el que se describen las actividades del sistema y de sus partes sobre la base de las interacciones de sus sub-sistemas en términos de objetivos concretos, de un programa. Esta distinción refiere a la de M. D. Mesarovic y D. P. Eckman (1961) entre aproximación "causal" y aproximación "teleológica."

Normalmente, se poseen suficientes informaciones respecto de un conjunto tecnológico para la medición de variables en el interior mismo del proceso sistémico. Simplemente, entonces se considera su estructura desde el ángulo, a) del montaje de componentes en serie, es decir de forma tal que la salida de uno es la entrada del siguiente, b) del montaje de componentes en paralelo, es decir de forma en que los componentes contribuye a la salida del conjunto. La siguiente estructuración es la más natural de un sistema : para un sub-sistema en serie, la avería de uno de los componentes conlleva la del sistema en su conjunto, y se remedia mediante un sub-sistema redundante en paralelo, en estos dos casos, no obstante se derivan leyes simples que entre la confiabilidad de los componentes; en representaciones de diagramas en bloque de la funciones de transferencia de un proceso pueden combinarse los pasos paralelos, y una relación simple une las uniones de los bloques en serie ; para encontrar soluciones para 
los cuellos de botella, debe resolverse un problema de filas de espera en cascada o múltiples...

Para iluminar la estructura funcional de un conjunto tecnológico complejo, debe partirse de un punto de vista menos físico, que es más habitual para psicólogos o ingenieros organizacionales. Los clivajes de algunas categorías se perciben en la globalidad del comportamiento del sistema. Tal como perfectamente lo subraya M. Merleau-Ponty (1942), "las estructuras a las que se llega de esta forma, no son, como aquellas del organismo, ni fenómenos parcelarios que las orientan, sino de ideas de las cuales participan sin por tanto contenerlas en sí." El sistema se comporta como un todo en la medida en que es posible introducir la noción de función mediante la atribución de un significado común a las diversas manifestaciones de la actividad de las partes del sistema. Cuando se analiza por ejemplo la interacción entre la función de producción y de la prevención (Faverge, 1967) el objetivo es comprender el comportamiento del hombre, unidad funcional de la organización. Este aspecto del estudio de los sistemas hombre-máquina es actualmente predominante, cualquiera sea por cierto la orientación particular de las investigaciones : que se compare por ejemplo a lo dicho por R. M. Gagné (1962) :

"Todo sistema, por poco que sea razonablemente complejo requiere una interacción real entre hombre y las partes del sistema que pueden ser máquinas, otros hombres o una combinación de ambos. Debe entonces encontrarse un modelo de pensamiento que se aplique a las funciones de las máquinas y a las funciones del hombre en el contexto de un marco que haga posible la puesta en relación de estos dos tipos de función y de objetivos comunes, es decir los objetivos del sistema" y D. I. Iordansky (1965) " los hombres, los equipos automatizados, las máquinas que son los elementos de grandes sistemas son las condiciones reales de las estructuras complejas y específicas cuyo estudio es, a todas pruebas, interesante (...)" y yendo aún más lejos, caracteriza este tipo de investigación, que se preocupa por el "aspecto estático y dinámico de estructuras funcionales y jerárquicas de grandes sistemas (...), de aspectos dinámicos de relaciones recíprocas entre los elementos humanos y máquinas, de relaciones mutuas entre miembros de colectividades al interior de sub-sistemas y entre colectividades de distintos sub-sistemas, entre superiores y subordinados (...)."

El componente primordial de la organización de un sistema, el conjunto de interacciones entre las diversas funciones de este, se representan mediante la interacción de tres términos. La producción de una salida acciona sobre la unidad operacional a partir de dos entradas, una provista de información de parte de la otra, y la otra caracterizada por "su programa," "su objetivo ("goal seeking unit" de M. D. Mesarovic y D. P. Eckman (1961)). Se observa que una unidad funcional o célula puede ser un elemento regulador, humano o mecánico, en este segundo caso, se la considerará como parte de lo que denominamos proceso.

\section{Estructura e historia}

30 Es evidente que la utilización del concepto de estructura incluye el tiempo como condición de su definición. La identificación de la estructura de un sistema depende de la existencia de lazos relativamente estables entre los elementos en un intervalo de tiempo determinado. La historia de un sistema es entonces la historia de su estructura, 
la pluralidad de sus rupturas de equilibrio, de modificaciones de proceso, del paso de una organización a otra.

Existen pocos estudios sobre la evolución histórica de sistemas tecnológicos complejos, o más bien existe sobre uno $u$ otro aspecto, técnico por ejemplo se cita en este marco la muy buena tesis de G. Simondon (1958) o económico pero no bajo el doble punto de vista de la evolución conjugada de la estructura operacional y de la estructura funcional de un conjunto tecnológico. Es entonces difícil ofrecer un repaso de los modelos históricos del estudio de los sistemas hombre-máquina, al menos en los términos propuestos, definiremos dos características de los sistemas adaptables, y por lo tanto reflejo de los cambios históricos, la flexibilidad y la mutabilidad.

La mutabilidad es una característica de la estructura funcional de un sistema abierto al ambiente y sumiso a sus presiones, que acepta la toma de decisiones en el contexto de las incertidumbres creadas por los espacios marginales que existen entre sí y el medio exterior (Faverge, 1966b). La traducción de esta mutabilidad en la estructura operacional es la flexibilidad, cualidad del proceso de un sistema mediante el cual las unidades funcionales facilitan la aplicación de cambios necesarios como resultado de fluctuaciones tanto económicas como tecnológicas.

\section{El sistema hombres-máquinas}

Desde la perspectiva de la ergonomía de los sistemas hombres-máquina, el lazo de causalidad recíproca, de implicación mutua que une el proceso tecnológico, en conjunto de relaciones entrada-salida entre unidades operacionales y su inventor, el hombre, no puede ignorarse; sería arbitrario separar la realidad humana cristalizada en las estructuras operacionales (lo que permite reconocer las funciones) y las unidades funcionales creadoras y organizadoras, los hombres que aseguran la supervivencia mediante el control, el mantenimiento, la coordinación, la adaptación.

34 Al momento de analizar un sistema, la estructura operacional no es sino una abstracción que se desprende de su aparato funcional, un dato inmediato que facilita la representación.

El hombre debe ser visto ya sea como un componente del proceso, como portador de herramientas, con un rol que se encuentra por debajo de su individualidad técnica (la evolución tecnológica tiende a liberarlo de estas tareas), o cómo un elemento de la organización de la estructura funcional del conjunto, y por tanto con un rol que va más allá de su individualidad técnica (Simondon, 1958). Desde esta perspectiva, es legítimo pretender que puesto que el hombre concibe las máquinas a partir de sus propias funciones que se produce inversamente la materialización de sus actividades, la máquina no es sino un modelo del hombre porque el hombre la construye a su imagen. EL mismo principio de reciprocidad lo hace percibir a través de la estructura funcional de un sistema un organismo vivo (Faverge, 1966b).

Estamos en este punto en condiciones de circunscribir el ámbito de las investigaciones sobre los sistemas hombres-máquinas, cómo el estudio de la conjunción de la estructura operacional y de la estructura funcional mediante las características globales del desempeño del sistema tales como la estabilidad, la fiabilidad, la recuperabilidad y de las características globales de su adaptación estructural, su 
flexibilidad y mutabilidad, lo que nos lleva en particular a interesarnos sobre la interacción entre el nivel de la organización y el nivel del proceso.

\section{BIBLIOGRAFÍA}

Ashby, W. R. (1947). Principles of the self-organizing system. J. Gen. Psych., 37, 125-128

Ashby, W. R. (1956). An introduction to cybernetics. London : Champan and Hall.

Ashby, W. R. (1961). Principles of the self-organizing system In Von Foerster H. \& Zopf G. W.

(eds.). Principles of Self-Organization, (1962) (pp. 255-278).

Beer, S. (1961). Toward the cybernetic factory. In Von Foerster H. \& Zopf G. W. (eds.). Principles of Self-Organization, (1962) (pp. 25-80).

Barlow, R. E. \& Proschan, F. (1965). Mathematical Theory of reliability. New York : Wiley.

Eckman, D. P. (ed.)(1961). Systems : Research and Design, Proc. Ist. Syst. Symp. Case Inst. Inst. Techn. (p.160,). New York : Wiley.

Faverge, J. M., et al. (1966a). L'ergonomie des processus industriels. Univ. Libre de Bruxelles : Ed. Inst. Sociol.

Faverge, J. M. (1966b). L'organizzazione viva. Riv. Psi. Lav., 4, 39-56

Faverge, J. M. (1967). Une analyse fonctionelle dualiste des activités des cellules d'un système. Revue Philosophique (sous presse),

Gagne, R. M. (1962). Human functions in systems. In Gagne R. M. (ed.). Psychological Principles in System Development (p. 65). Holt.

Goldman, A. S. y Slattery, T. B. (1964). Maintainability : a major element of system effectiveness. New York : Wiley.

Goldstein, K. (1934). Der Aufbay des organismus. La Haye : M. Nijhoff..

Iordansky, D. I. (1963). Nékotoryé zadatchi issledovania kollektikov lioudei, outchastvouioutchikh v oupravlénii bolchimi sistemami. In D. A. Ochanine (réd.), Sistema "tchlovek i automat" (pp. 20-27). Moscú : Naouka, 1965.

Mach, E. (1910). Ueber das Prinzip der Vergleichung in der Physik. Popular-Wissenchafliche Vorlesungen, Leipzig.

Meadows, P. (1957). Models, systems and science. Amer. Soc. Rev., 22 (3).

Merleau-Ponty, M. (1942). La structure du comportement. Paris : P.U.F.

Mesarovic, M. D. \& Eckman, D. P. (1961). On some basic concepts of General Systems Theory, Proc. IIId Int. Cong. Cybern., Namur, Ass. Int. Cybern, 1965.

Mesarovic, M. D. (1963). Foundations for a General Systems Theory. In, M.D. Mesarovic (ed.), Views on General Systems Theory (pp. 1-24), $1964 .$. 
M.D. Mesarovic (ed.) (1964). Views on General Systems Theory. Proc. IId Systems Symp., Case Inst. Techn.. Wiley : New York.

Miller, J. G. (1965). Living systems : Basic Concepts, Structure and Process, Cross Level Hypothesis. Behav. Sc., 10 (3, 4), 193-237 \& 334-411.

Morton, J. A. (1959). Integration of Systems Ingineering with Component Development. Electr.Manuf., 64 (85).

Pask, G. (1959). The natural history of Networks. In Yovits M. C. y Cameron S. (eds) Self-Organizing Systems, (1959).

Simondon, G. (1958). Du mode d'existence des objets techniques. Paris : Aubier.

Timpe, K. P. (1966). Critères d'optimisation dans le système homme-machine. Act. XVIIIè Cong. Int. Psych., Moscú.

Von Bertalanffy, L. (1945). Zu einer allgemeinen sytemlehre, Bl. Deutsche Philos., 18, publicado en Biologia Generalis, 19, 1949, 114-129.

Von Bertalanffy, L. (1949). Das Bioloscho Weltbild. Berne : A. Francke.

Von Bertalanffy, L. (1950a). An outline of General System Theory. Br. J. Philos. Sci. 1, 134-165.

Von Bertalanffy, L. (1950b). The theory of open systems in physics and biology. Science, 111, 23-29.

Von Foerster, H. \& Zopf, G. W. (eds.)(1962). Principles of self-Organizations. Trans. Univ. Illinois. Symposium on Self-Organization, 1961. Cambridge : Pergamon Press :.

Yovits, M. C. \& Cameron, S. (eds) (1960). Self-Organizing Systems, Interdisciplinary Conf. Illinois, 1959. Cambridge : Pergamon Press.

\section{RESÚMENES}

La rapidez del progreso técnico y el tamaño de los conjuntos tecnológicos actuales exigen que el ergónomo se esfuerce por superar estudios tradicionales del puesto que se limitan en el tiempo y el espacio. Desde esta perspectiva, pareciera ser interesante introducir la noción de "sistemas hombre-máquina" y para ello utilizar el aporte conceptual del movimiento de estudio de sistemas : teoría general de sistemas, teorías de sistemas de auto-organización y tecnología de sistemas. Tras una revisión esquemática de estos, el autor presenta las características generales de un sistema e intenta circunscribir el ámbito particular de las investigaciones sobre sistemas hombre-máquina mediante la distinción del nivel de proceso y del nivel de organización de un sistema.

A rapidez do progresso técnico e a grandiosidade dos conjuntos tecnológicos actuais exigem do ergónomo um esforço de ultrapassagem do tradicional estudo de posto limitado no espaço e no tempo. Nesta perspectiva parece que seria interessante introduzir a noção de "sistema-homemmáquina » e para isso utilizar o contributo conceptual dos movimentos de estudo de sistemas: teoria geral dos sistemas, teorias dos sistemas auto-organizados e tecnologia dos sistemas. Após uma apreciação sumária dos mesmos o autor evoca algumas características muito gerais de um sistema e tenta a seguir circunscrever o domínio da pesquisa sobre os sistemas homem-máquina em particular graças à distinção entre o nível do processo e o nível da organização de um sistema.

La rapidité du progrès technique et la grandeur des ensembles technologiques actuels exigent de l'ergonome un effort de dépassement de la traditionnelle étude de poste limitée dans le temps et dans l'espace. Dans cette perspective, il semble qu'il y aurait intérêt à introduire la notion de 
“système-hommes-machines » et pour cela utiliser l'apport conceptuel des mouvements d'étude de systèmes: théorie générale des systèmes, théories des systèmes auto-organisation et technologie des systèmes. Après un aperçu schématique de ceux-ci l'auteur envisage quelques caractéristiques très générales d'un système et tente ensuite de circonscrire le domaine de recherches sur les systèmes hommes-machines en particulier grâce à la distinction entre le niveau du processus et le niveau de l'organisation d'un système.

The speed of technical progress and the size of the present technological systems require from the ergonomist an effort to go beyond the traditional job analysis, limited in time and space. From this view point it should be interesting to bring in the concept of "man-machine systems" and to use for this purpose the conceptual contributions of the different branches of study that deal with systems: general systems theory, theory of self-organizing systems and systems engineering. After a schematic description of these contributions the author first examines some very general characteristics of a system and tries to limit the field research about man-machine systems, especially by making a distinction between the process level and thee organizations level of a system.

\section{AUTORES}

\section{MICHEL OLIVIER}

Laboratoire de Psychologie de l'Université Libre de Bruxelles 